\title{
Chapter 17 \\ Near Infrared Light Scattering Changes Following Acute Brain Injury
}

\author{
David Highton, Ilias Tachtsidis, Alison Tucker, Clare Elwell, \\ and Martin Smith
}

\begin{abstract}
Acute brain injury (ABI) is associated with changes in near infrared light absorption reflecting haemodynamic and metabolic status via changes in cerebral oxygenation (haemoglobin oxygenation and cytochrome-c-oxidase oxidation). Light scattering has not been comprehensively investigated following ABI and may be an important confounding factor in the assessment of chromophore concentration changes, and/or a novel non-invasive optical marker of brain tissue morphology, cytostructure, hence metabolic status. The aim of this study is to characterize light scattering following adult ABI. Time resolved spectroscopy was performed as a component of multimodal neuromonitoring in critically ill brain injured patients. The scattering coefficient $\left(\mu_{s}^{\prime}\right)$, absorption coefficient and cerebral haemoglobin oxygen saturation $\left(\mathrm{SO}_{2}\right)$ were derived by fitting the time resolved data. Cerebral infarction was subsequently defined on routine clinical imaging. In total, 21 patients with $\mathrm{ABI}$ were studied. Ten patients suffered a unilateral frontal infarction, and mean $\mu_{s}^{\prime}$ was lower over infarcted compared to non-infarcted cortex (injured $6.9 / \mathrm{cm}$, non-injured $8.2 / \mathrm{cm} \mathrm{p}=0.002$ ). $\mathrm{SO}_{2}$ did not differ significantly between the two sides (injured $69.3 \%$, non-injured $69.0 \% \mathrm{p}=0.7$ ). Cerebral infarction is associated with changes in $\mu_{s}^{\prime}$ which might be a novel marker of cerebral injury and will interfere
\end{abstract}

This chapter was originally published under a CC BY-NC 4.0 license, but has now been made available under a CC BY 4.0 license. An erratum to this chapter can be found at DOI 10.1007/978-1-4939-3023-4_66.

D. Highton $(\bowtie)$

Neurocritical Care, University College Hospitals, Queen Square, London, UK e-mail: d.highton@ucl.ac.uk

I. Tachtsidis • A. Tucker

Medical Physics and Bioengineering, University College London, Malet Place, London, UK

C. Elwell

Department of Medical Physics and Biomedical Engineering, University College London,

London, UK

M. Smith

Neurocritical Care, University College Hospitals, Queen Square, London, UK

Medical Physics and Bioengineering, University College London, Malet Place, London, UK

NIHR University College London Hospitals Biomedical Research Centre, London, UK

(C) The Author(s) 2016

C.E. Elwell et al. (eds.), Oxygen Transport to Tissue XXXVII, Advances in

Experimental Medicine and Biology 876, DOI 10.1007/978-1-4939-3023-4_17 
with quantification of haemoglobin/cytochrome c oxidase concentration. Although further work combining optical and physiological analysis is required to elucidate the significance of these results, $\mu_{s}^{\prime}$ may be uniquely placed as a non-invasive biomarker of cerebral energy failure as well as gross tissue changes.

Keywords Near infrared spectroscopy - Brain injury - Scattering - Cerebral ischaemia

\section{Introduction}

The optical characteristics of cerebral tissues following acute brain injury (ABI) have been of considerable clinical interest, because they can be exploited to interrogate cerebral oxygenation in-vivo, non-invasively. An optical window in the near infrared spectrum $(700-900 \mathrm{~nm})$ facilitates measurement of the dominant absorbing chromophores in this region—oxy/deoxy-haemoglobin and cytochrome c oxidase, by their relative specific absorption spectra - thus inferring information about the haemodynamic and metabolic status of the brain [1]. However light transport through complex biological media is highly dependent on light scattering as well as absorption, which has not been comprehensively investigated following $\mathrm{ABI}$, and may be an important confounding factor in the assessment of chromophore concentration changes [2], and/or a novel non-invasive optical marker of brain tissue morphology, cytostructure, hence metabolic status [3]. The aim of this study is to characterize light scattering in brain tissue following adult ABI.

\section{Methods}

A total of 21 critically ill, ventilated brain injured patients were recruited following ethical approval and representative consent. Time resolved spectroscopy (TRS-20, Hamamatsu Photonics KK) was performed as a component of multimodal neuromonitoring in critically ill brain injured patients. Serial recordings were taken whilst in critical care. The reduced scattering coefficient $\left(\mu_{s}^{\prime}\right)$, absorption coefficient, cerebral haemoglobin oxygen saturation $\left(\mathrm{SO}_{2}\right)$ and total haemoglobin concentration $([\mathrm{HbT}])$ were derived by fitting the time resolved data (diffusion equation for light transport in a semi-infinite homogeneous medium, fitting the entire temporal point spread function, as standard within the Hamamatsu software). Three bilateral time-resolved recordings were made over frontal cortex at $4 \mathrm{~cm}$ source detector separation with a $5 \mathrm{~s}$ acquisition time. The mean of these three recordings were used for comparison. Cerebral infarction was subsequently defined on routine clinical imaging and paired comparison of $\mu_{s}^{\prime}$ was performed in patients with unilateral infarction, using the paired $t$-test. $\mu_{s}^{\prime}$

The wavelength dependence of $\mu_{s}^{\prime}$ was analysed using least squares regression from the group data, across the three wavelengths measured (761, 801 and $834 \mathrm{~nm}$ ), the $95 \%$ confidence intervals and F-statistic is reported. To facilitate comparison with the literature three previously described models were fitted: $\mu_{s}^{\prime}(\lambda)=a \lambda+b$, 
$\mu_{s}(\lambda)=b \lambda^{-a}$ normalized to the mean scattering value at $801 \mathrm{~nm}$ as in Matcher et al. [4], and $\mu_{s}^{\prime}(\lambda)=b(\lambda / 500 \mathrm{~nm})^{-a}$ from Jacques et al. [5].

\section{Results}

Patients' characteristics are summarised in Table 17.1 and the $\mu_{s}^{\prime}$ and $\mathrm{SO}_{2}$ data are summarised in Fig. 17.1. Ten patients suffered a unilateral frontal infarction, and mean $\mu_{s}^{\prime}$ was lower over infarcted compared to non-infarcted cortex (Table 17.2). $\mathrm{SO}_{2}$ (injured $69.3 \%$, non-injured $69.0 \% \mathrm{p}=0.7$ ) and $\left[\mathrm{HbT}\right.$ ] (injured $64.6 \mu \mathrm{mol}^{-1}$, non-injured $\left.51.9 \mu \mathrm{mol}^{-1} \mathrm{p}=0.09\right)$ did not differ significantly. The time course of $\mu_{s}^{\prime}$ data is shown in Fig. 17.1 suggesting a trend of increasing $\mu_{s}^{\prime}$ with respect to time in the infarct group:- however the patient group is heterogeneous, as is the onset of infarction.

The wavelength dependence of $\mu_{s}^{\prime}$ and fitted parameters are shown in Table 17.3. Using the linear model there was no significant difference in wavelength dependence defined by parameter $a$ (injured $-9.3 \times 10^{-3}$, non-injured $-9.2 \times 10^{-3} \mathrm{p}=0.88$ )

\section{Conclusions}

Cerebral infarction is associated with significant reduction in $\mu_{s}^{\prime}$ below previously reported values for normal adults [4]. Unilateral infarction was not associated with a similar difference in $\mathrm{SO}_{2}$ or $[\mathrm{HbT}]$ indicating either less discriminatory ability of these complex physiological variables or error in the assumptions underlying their derivation. The wavelength dependence of $\mu_{s}^{\prime}$ was approximately linear over the narrow band of wavelengths studied and revealed similar parameter estimates to other reports of cerebral tissues in vivo [5] and in vitro [6]. This did not vary between injured and non-injured cortex. Clearly these findings have potentially important implications for analysis using differential spectroscopy over injured brain as variation in $\mu_{s}^{\prime}$ violates assumptions required to calculate concentration changes.

The physiological origin of $\mu_{s}^{\prime}$ reduction is also of considerable interest. Studies on exposed or in vitro cerebral cortex identify subcellular structures as the dominant scatterers in the near infrared spectrum with the mitochondria as a key contributor-given its typical size of 500-1000 nm. Thus scattering has been used experimentally to define mitochondrial volume and density-hence metabolic compromise [3]. Translation of this paradigm into the clinic might hold considerable promise, but it is important to consider the differences in measurement

Table 17.1 Patient characteristics. Reported as median [quartile] or number (percentage)

\begin{tabular}{l|l}
\hline Age (years) & $58[47,70]$ \\
\hline Diagnosis & \\
\hline Subarachnoid haemorrhage (\%) & $11(52)$ \\
\hline Intracerebral haemorrhage (\%) & $8(38)$ \\
\hline Ischaemic stroke (\%) & $1(5)$ \\
\hline Traumatic brain injury (\%) & $1(5)$ \\
\hline In-hospital mortality (\%) & $4(19)$ \\
\hline
\end{tabular}



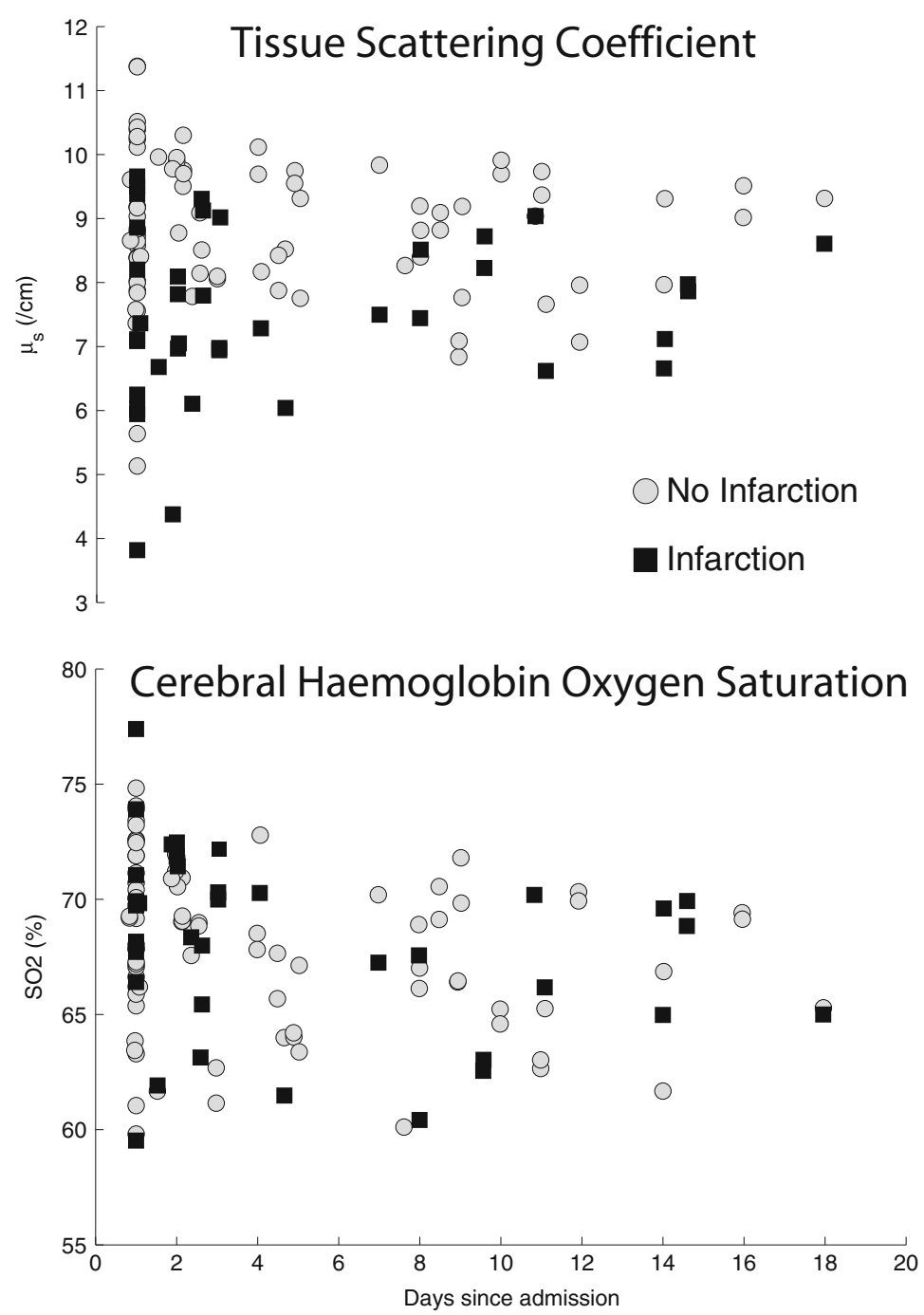

Fig. 17.1 Mean $\mu_{s}^{\prime}(834 \mathrm{~nm})$ and $\mathrm{SO}_{2}$ for each day post intensive care admission in cerebral hemispheres demonstrating infarction versus no infarction. The lower scattering in infarcted cerebral hemisphere is evident, whereas there is no appreciable pattern with $\mathrm{SO}_{2}$

Table 17.2 $\mu_{s}^{\prime}$ measured over infarcted and non-infarcted cortex

\begin{tabular}{l|l|l|l}
\hline & $\mu_{s}^{\prime} 761 \mathrm{~nm}\left(\mathrm{~cm}^{-1}\right)$ & $\mu_{s}^{\prime} 801 \mathrm{~nm}\left(\mathrm{~cm}^{-1}\right)$ & $\mu_{s}^{\prime} 834 \mathrm{~nm}\left(\mathrm{~cm}^{-1}\right)$ \\
\hline Infarct & $7.43(1.74)$ & $7.18(1.64)$ & $6.87(1.57)$ \\
\hline No infarct & $8.84(1.51)$ & $8.52(1.45)$ & $8.20(1.43)$ \\
\hline p-value & 0.003 & 0.003 & 0.002 \\
\hline
\end{tabular}

Brackets denote standard deviation 
Table 17.3 Wavelength dependence of $\mu_{s}^{\prime}$ fitted to three established models

\begin{tabular}{l|l|l|l}
\hline & $\mu_{s}^{\prime}(\lambda)=\mathrm{a} \lambda+\mathrm{b}$ & $\mu_{s}^{\prime}(\lambda)=\mathrm{b} \lambda^{-\mathrm{a}}$ & $\mu_{s}^{\prime}(\lambda)=\mathrm{b}(\lambda / 500 \mathrm{~nm})^{-\mathrm{a}}$ \\
\hline $\mathrm{a}\left(\mathrm{cm}^{-1} \mathrm{~nm}^{-1}\right)$ & $\begin{array}{l}-0.0085\left(-9.0 \times 10^{-3}\right. \\
\left.\text { to }-8.1 \times 10^{-3}\right)\end{array}$ & $0.77(0.73-0.81)$ & $0.77(0.31-1.22)$ \\
\hline $\mathrm{b}\left(\mathrm{cm}^{-1} \mathrm{~nm}^{-1}\right)$ & $15.6(15.3-16.0)$ & $1485(1134-1942)$ & $12.4(10.1-12.5)$ \\
\hline F-statistic & 1442 & 1424 & 11.14 \\
\hline p-value & $<0.0001$ & $<0.0001$ & 0.001 \\
\hline
\end{tabular}

Brackets denote the $95 \%$ confidence limits of the model parameters. All models fit the data significantly

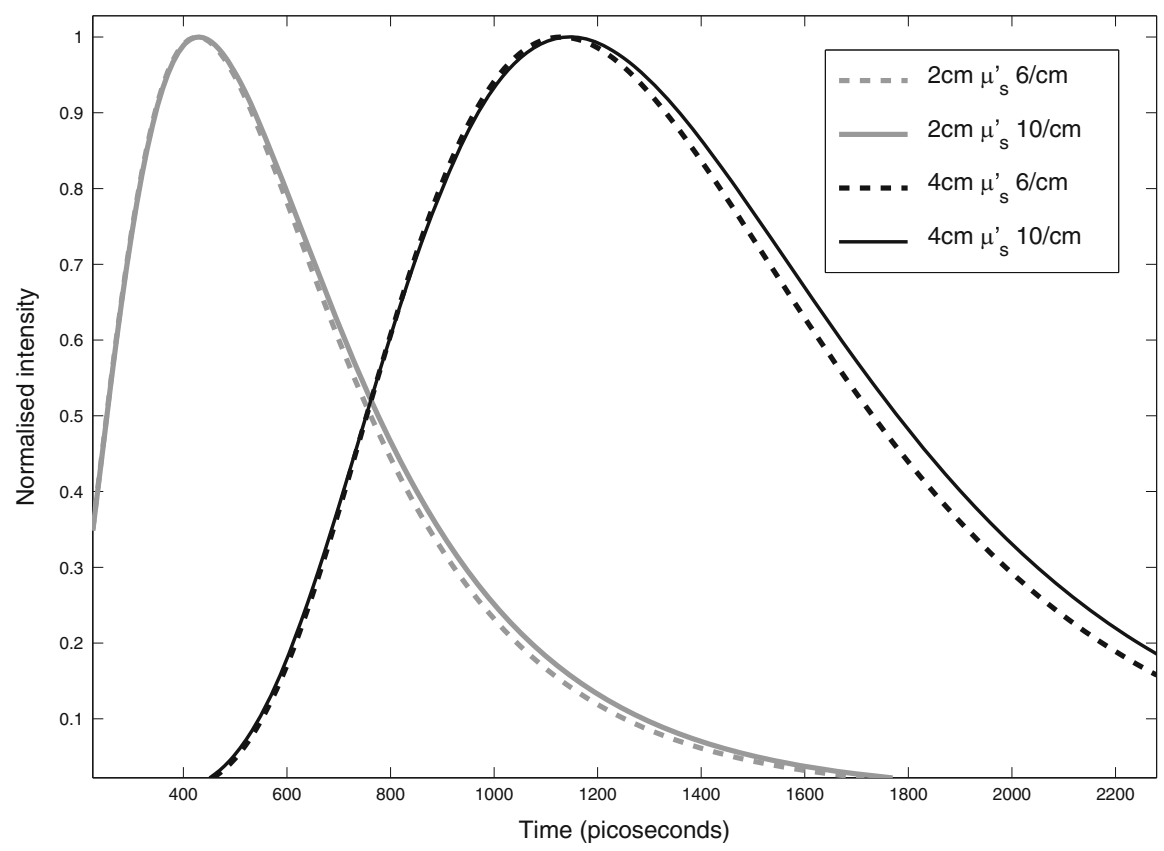

Fig. 17.2 Two-layer model simulation of the effect of cerebral $\mu_{s}^{\prime}$ on TPSF data at source detector separations of 2 and $4 \mathrm{~cm}$. It can be seen that the TPSF has much greater sensitivity to $\mu_{s}^{\prime}$ changes in the deep layer at $4 \mathrm{~cm}$ source detector separation than $2 \mathrm{~cm}$. A two-layer semi-infinite slab geometry was used in this simulation, layer $1\left(1 \mathrm{~cm}\right.$ depth, $\mu_{a} 0.1 / \mathrm{cm}$ and $\left.\mu_{s}^{\prime} 10 / \mathrm{cm}\right)$, layer $2(5 \mathrm{~cm}$ depth, $\mu_{a} 0.1 / \mathrm{cm}$ and $\mu_{s}^{\prime} 6 / \mathrm{cm}$ or $10 / \mathrm{cm}$ ) using a model from Liemert et al. [8]

technique and underlying assumptions fitting the time resolved data. Specifically fitting the time resolved temporal point spread function (TPSF) assuming a homogenous semi-infinite medium may be an oversimplification of cerebral structure following brain injury and especially after surgical intervention. A specific issue which has been discussed is the sensitivity of the TPSF to $\mu_{s}^{\prime}$ changes in the brain, rather than overlying extracerebral tissues. Simulated photon diffusion considering a two-layer structure and $2 \mathrm{~cm}$ source detector separation predicts little sensitivity in the TPSF to $\mu_{s}^{\prime}$ below $1 \mathrm{~cm}$ depth [7] implying that much of our findings could be superficial tissue changes. However repeating simulations in a simple two-layer slab geometry shows greater sensitivity of the TPSF to changes in scattering to the $4 \mathrm{~cm}$ source detector separation we have used (Fig. 17.2). 
Further work is required to identify the physiological significance of our findings. Individualized optical modeling based on anatomical data from radiological imaging is feasible, and may define the optical situation with further clarity. Combining optical and physiological modeling may hold the key to elucidate individualized physiological explanations for our multimodal optical and physiological data. Thus optical scattering might have considerable potential as a novel independent optical signal of cerebral ischaemia/infarction following ABI, by characterising the resultant changes in mitochondrial morphology and cellular structure in vivo — an approach which appears more discriminatory than traditional haemoglobin based measures.

Acknowledgments This work was supported by the Wellcome Trust undertaken at University College London Hospitals and partially funded by the Department of Health's National Institute for Health Research Centres funding scheme via the UCLH/UCL Biomedical Research Centre. The authors are indebted to the medical and nursing staff of the Neurocritical Care Unit at the National Hospital for Neurology \& Neurosurgery, and to the study patients and their families. We would like to thank Hamamatsu Photonics for the loan of their spectrometer.

\section{References}

1. Ghosh A, Elwell CE, Smith M (2012) Review article: cerebral near-infrared spectroscopy in adults: a work in progress. Anesth Analg 115:1373-1383

2. Metz AJ, Biallas M, Jenny C et al (2013) The effect of basic assumptions on the tissue oxygen saturation value of near infrared spectroscopy. Adv Exp Med Biol 765:169-175

3. Johnson LJ, Chung W, Hanley DF et al (2002) Optical scatter imaging detects mitochondrial swelling in living tissue slices. Neuroimage 17:1649-1657

4. Matcher SJ, Cope M, Delpy DT (1997) In vivo measurements of the wavelength dependence of tissue-scattering coefficients between 760 and $900 \mathrm{~nm}$ measured with time-resolved spectroscopy. Appl Opt 36:386-396

5. Torricelli A, Pifferi A, Taroni P et al (2001) In vivo optical characterization of human tissues from 610 to $1010 \mathrm{~nm}$ by time-resolved reflectance spectroscopy. Phys Med Biol 46:2227-2237

6. Jacques SJ (2013) Optical properties of biological tissues: a review. Phys Med Biol 58:R37-R61

7. Selb J, Ogden TM, Dubb J, Fang Q et al (2014) Comparison of a layered slab and an atlas head model for Monte Carlo fitting of time-domain near-infrared spectroscopy data of the adult head. J Biomed Opt 19:16010-016010

8. Liemert A, Kienle A (2010) Light diffusion in N-layered turbid media: frequency and time domains. J Biomed Opt 15:025002

Open Access This chapter is licensed under the terms of the Creative Commons Attribution 4.0 International License (http://creativecommons.org/licenses/by/4.0/), which permits use, sharing, adaptation, distribution and reproduction in any medium or format, as long as you give appropriate credit to the original author(s) and the source, provide a link to the Creative Commons license and indicate if changes were made.

The images or other third party material in this chapter are included in the chapter's Creative Commons license, unless indicated otherwise in a credit line to the material. If material is not included in the chapter's Creative Commons license and your intended use is not permitted by statutory regulation or exceeds the permitted use, you will need to obtain permission directly from the copyright holder.

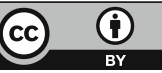

